\title{
Consumers' Willingness to Buy Brand Ginseng Products and its Empirical Study on the Influence Factors
}

\author{
Yundi Dai ${ }^{12, \text { a }}$, Yanjiao Wen ${ }^{2, a}$, Wei Xie ${ }^{2, a}$, Huimin $\mathrm{Li}^{2, a}$ \\ ${ }^{1}$ College of Economics and Management, Jilin Agricultural University, Changchun, 130118, China \\ ${ }^{2}$ Changchun College of Finance and Economics, Department of Management, Changchun, 130000, \\ China \\ aemail: 1013889886@qq.com
}

Keywords: Brand ginseng products; Purchase intention; Influencing factors; Empirical analysis

\begin{abstract}
This Logistic model on consumers' willingness to buy brand ginseng products and measurement analysis of influencing factors. Results show that consumers buy brands of ginseng products under the influence of a variety of factors, the primary factor for the consumer's personal factors, including consumer's gender, age, marital status, level of education, household size, household income, prices, purchases, etc.; followed by the awareness of consumers. This article will from three aspects of ginseng to build brand strategy system.
\end{abstract}

\section{Introduction}

For the same products and different brand, different consumer awareness is also difference, resulting in different buying behavior. Ginseng brand building is that from different sides of enterprise, consumer, market and government, people considers the ginseng enterprise about the brand investment and the use of brand result as well as the brand benefit received by consumers. Ginseng brand products and brand-name products are high quality, high-grade symbol and embodiment, brand culture brings the consumer spirit income, consumption level enhancement and the widening income gap. Ginseng product brand provides great realistic and potential markets on consumers, and ginseng brand building is arranged to meet consumer demand.

The implementation of brand strategy is to optimize the allocation of ginseng resources, scientific and technological innovation and sustainable development [1]. Seizing the opportunity and integrating the regional brand, let these regional brands do a joint, it is not only to avoid the waste of resources, but also is better with human resources [2]. With the improvement of people's living standards, self-health care awareness and the rapid development of industry, domestic demand quantity of ginseng products will continue to grow. Ginseng product consumer is the ginseng industry chain terminal, but also is the starting point of modern ginseng industry system as consumer oriented. Study on consumption of ginseng products has great significance for the development of ginseng industry guide [3].

Brand construction focuses on the relationship between brand and consumers. If the product brand has no value on consumers, it would be meaningless on the enterprise. Famous brand expert Keller in 1993 defined the brand value, he said that the consumer responded to brand cognition produced by a brand of all marketing activities. Lasser, Mittal and Sharma in 1995 believed that brand value was attached to the product brand name, and it can bring the additional benefits and cognitive utility on consumers. At present, China Jilin "Changbai Mountain Ginseng" brand has been launched, it has achieved competitive position in South Korea "Guan Zhuang” and other sustainable development of well-known brands, and we need to establish a set of brand strategy system [4]. With a unique geographical advantage and long history inheritance based, people should train a group of good industrial foundation, but also have a greater influence and regional brand in the market reputation, which has become the important strategic weapons of regional economic development and the regional competitiveness promotion [5].

Based on consumer survey data, this paper mainly uses the logistic model to empirical analyze the ginseng product brand consumption intention and its influencing factors. The results can be for 
Jilin province government make ginseng brand building strategy provides the reference.

\section{Theoretical Framework and Research Hypothesis}

Theoretical Framework. The brand ginseng products are resources and the result of increased input in quality, service, food safety and other aspects of the advantages, so it has relatively high production cost [6-8]. For example, the brand ginseng inputs is more time and labor inputs, the use of limiting pesticides and fertilizers reduces production to certain extent, so the production needs of brand ginseng is relatively high price to support [9]. Consumers are often reluctant to pay for high quality products too much cost, but with the growth of national income and improve of education level, and people's purchase will have a trend of increasing.

Through the results of the questionnaire, the authors carryout statistical analysis and study the influence factors of consumers to buy brand ginseng behavior, which provide a theoretical basis for the healthy and orderly development of ginseng industry in china.

The linear regression model is more commonly used model in the application of quantitative analysis. However, in many cases, the linear regression model is encountered, for example when the dependent variable is a categorical variable and is not a continuous variable, linear regression will not be applied [10-12]. In the analysis of categorical variables, statistical method is commonly used in the log linear model, and logistic regression model is a special way of log linear model. This paper uses two logistic regression models to analyze the data, establishing the model mathematical expressions as follows:

$$
\ln (p)=f\left(x_{i}\right)+\varepsilon_{i}
$$

The introduction of related factors in qualitative analysis, the equation expression of specific mathematical model is:

$\ln (p)=f$ (sex, age, ms, edu, income, family, purpose, price, $Q S$, consume, brand)

Among them, $P$ represents the dependent variable, sex represents the sample consumer gender, age represents sample consumer age, edu represents the education level of sample consumer, income represents the family income of sample consumer, family represents the number of the family population of sample consumer, and purpose represents the personal purpose of consumers buy. The $Q S$ represents whether sample consumers understand the quality certification marks, the consume represents the amount of the sample consumers' annual consumption ginseng products, the brand represents whether sample consumers understand the brand ginseng. The dependent variables are handled as follows: buying brand ginseng is 1 , and no-buying brand ginseng is 0 . The quantification process of affecting factor index is shown in table 1. 
Table.1 The description of independent variable value

\begin{tabular}{|c|l|}
\hline $\begin{array}{c}\text { Influence factors } \\
\text { Consumer personal factors }\end{array}$ & \multicolumn{1}{|c|}{ Quantitative approach } \\
\hline sex & Consumers are male, then it is 0; on contrast, it is 1. \\
\hline age & The age of consumers \\
\hline Ts & $\begin{array}{l}\text { The consumer is married, then it is 0; on contrast, unmarried is } \\
1 .\end{array}$ \\
\hline edu & $\begin{array}{l}\text { Consumer education: junior middle school and below is 1, high } \\
\text { school is 2, college is 3, undergraduate is 4, master's degree } \\
\text { and above are 5 }\end{array}$ \\
\hline income & The consumer's monthly family income (Yuan) \\
\hline family & The number of family consumers \\
\hline purpose & The personal purchase use is 0, and sending people is 1. \\
\hline price & Concerned about price is 1; on contrast, it is 0. \\
\hline consume & The average annual personal consumption amount (Yuan) \\
\hline Consumer cognition & Understanding the ginseng brand is 1; on contrast, it is 1. \\
\hline brand & $\begin{array}{l}\text { Understanding the quality certification marks is 1; on contrast, } \\
\text { it is 0. }\end{array}$ \\
\hline QS &
\end{tabular}

Research hypothesis. The study of domestic scholars generally believe that for the awareness of brand, the consumer is lower with the increase of age, increases with the improvement of education level and income growth, and the change of cognitive level will affect the consumer's purchase behavior. At the same time, consumer also affects the purchase behavior of ginseng brand for the ginseng quality security and other aspects.

\section{(1) The personal factors of consumers}

Personal characteristics and conditions will affect the consumer purchase behavior of brand ginseng. The consumer's personal factors include gender, age, marital status, education level, family size and family income and so on. From the gender perspective, women are much more contact in business information, so they have a deep understanding for the brand ginseng, and they are more likely to buy the brand ginseng. From the age, older people pay more attention to nutrition and health, and they are more likely to buy brand ginseng. From the point of the marriage condition, compared with single consumer, wedding consumers are more likely to buy the brand ginseng or visit the elders. From the point of education, higher education consumers have higher demands on the products' quality, safety, service and so on, and they are more inclined to the consumer brand ginseng. From the number of family, family members are more consumers, they buy brand ginseng to eat more likely. From the point of family income, family income is higher for consumers, and the consumer brand ginseng is more. From the price of consumer brand attitude, if consumers are more concerned about product prices, the possibility of the consumer brand ginseng is less; if consumers don't worry about the price of a product, the possibility of consumer brand ginseng is larger. From the consumer to buy the ginseng consumption amount, consumers buy brand ginseng as a gift, they are more likely to buy brand ginseng; on the contrary, they are small.

\section{(2) The consumer awareness for the brand ginseng}

Consumer includes many aspects for the awareness of the brand. In this paper, consumers know the ginseng brand as a measure index of consumer ginseng brand cognition, to measure consumer brand purchase behavior of ginseng. For consumers, they more understand brand quality, safety, health effects and nutrients, and the possibility of buying brand ginseng is more; on the other hand, the possibility of buying brand ginseng is small. At the same time, whether the consumers understand the quality certification marks, it also affects the purchase ginseng brand factors.

Through the qualitative analysis, and referring to the research experience of scholars, this paper proposes the hypotheses as follows:

Hypothesis one: consumer personal factors have impact on the purchase of brand ginseng. The 
consumer's personal factors include consumer's gender, age, marital status, education level, family income, family population, care about the extent of price, the annual consumption amount and the application of the purchase.

Hypothesis two: the degree of consumer awareness has impact on the purchase of brand ginseng. This paper will use whether consumers understand the ginseng brand and quality certification marks, to reflect consumer awareness of the brand ginseng.

\section{Data Sources and Sample Description}

For the study of consumer on the awareness and buying behavior of the brand ginseng, the author designed the questionnaire to survey research. This study used data from the Jilin agricultural university agriculture undergraduate association and some Jilin agricultural university graduate students. In July 2015 until October, the author caught out consumer survey in Changchun, Fusong, Tonghua, Baishan, Yanbian area. In 2015, the south students investigated on south market during winter and summer vacation, and they provide information for the Jilin province ginseng office. Respondents were the ginseng products distribution shop or ginseng professional market customers, the survey content related to the basic situation of respondent family, income, buyers on ginseng products cognition, purchase intention on brand ginseng product and other information. According to the survey data, the author issued a total of 550 questionnaires, in which the field survey had 450 copies, there were 389 questionnaires recovered, the check was 48 from a sample of excluding missing key variables data, 441 valid samples were efficiency reached, and the questionnaire efficiency was reached $90.1 \%$. The survey sample was mainly concentrated in the Changchun area of Jilin province, and this was mainly big city due to the investigation convenience and ginseng product consumer market.

Table.2 Variable statistic description

\begin{tabular}{|c|c|c|c|c|c|c|}
\hline & $N$ & Minimum & Maximum & \multicolumn{2}{|c|}{ Mean } & Std. Deviation \\
\hline & Statistic & Statistic & Statistic & Statistic & Std. Error & Statistic \\
\hline sex & 441 & 0 & 1.00 & 0.5079 & 0.02383 & 0.50050 \\
\hline age & 441 & 16.00 & 76.00 & 35.7937 & 0.57394 & 12.05275 \\
\hline ms & 441 & 0 & 1.00 & 0.3810 & 0.02315 & 0.48617 \\
\hline edu & 441 & 1.00 & 5.00 & 3.9070 & 0.04501 & 0.94531 \\
\hline income & 441 & 1007.00 & 13994.00 & $5.9149 \mathrm{E} 3$ & $1.48034 \mathrm{E} 2$ & 3108.70661 \\
\hline family & 441 & 1.00 & 9.00 & 3.3605 & 0.05085 & 1.06779 \\
\hline purpose & 441 & 0 & 1.00 & 0.5556 & 0.02369 & 0.49747 \\
\hline price & 441 & 0 & 1.00 & 0.0816 & 0.01305 & 0.27412 \\
\hline$Q S$ & 441 & 0 & 1.00 & 0.4286 & 0.02359 & 0.49543 \\
\hline consume & 441 & 0 & 10000.00 & $5.9879 \mathrm{E} 2$ & 41.57419 & 873.05802 \\
\hline brand & 441 & 0 & 1.00 & 0.4512 & 0.02372 & 0.49818 \\
\hline buy & 441 & 0 & 1.00 & 0.3696 & 0.02301 & 0.48325 \\
\hline
\end{tabular}

Data source: software output

As shown in table 2, it can be seen that the proportion of male and female respondents is average, in which the youngest consumers are at the age of 16, the maximum consumers are 76 years, and their mean age is in 35 years or so, the individual differences are larger. Marital status is in the majority of married, accounting for $62 \%$, unmarried accounted for $38 \%$, the level of average 
education is closed to undergraduate, and the individual differences are also larger. The number of family members is in the 1-9 range, and the average is 3 . The proportion of the purchase ginseng and the use of ginseng is basically the same. More than $90 \%$ people care about the product price. Consumers in the consumption amount of ginseng are in the $0-10000$ Yuan, and an average is 599 Yuan. More than $40 \%$ of consumers understand the ginseng brand and quality safety.

\section{Empirical Analysis Results and Discussion}

Regression analysis was carried out by two Yuan logistic model on consumer purchase brand ginseng behavior. The results are shown in table 3.

Table. 3 Model regression results

\begin{tabular}{|c|c|c|c|c|}
\hline Explanatory variables & $B$ & S.E. & Sig. & $\operatorname{Exp}(B)$ \\
\hline sex & -0.097 & 0.549 & 0.859 & 0.907 \\
\hline age & 0.182 & 0.032 & 00.000 & 0.833 \\
\hline$m s$ & -1.101 & 0.568 & 0.053 & 0.333 \\
\hline edu & 0.790 & 0.293 & 0.007 & 2.203 \\
\hline income & 0.001 & 0.000 & 0.000 & 0.999 \\
\hline family & 0.271 & .293 & 0.355 & 1.311 \\
\hline purpose & 0.322 & 0.580 & 0.579 & 1.380 \\
\hline price & -1.634 & 1.004 & 0.104 & 0.195 \\
\hline$Q S$ & 1.254 & .550 & 0.022 & 3.506 \\
\hline consume & 0.009 & 0.002 & 0.000 & 0.991 \\
\hline brand & 2.961 & 0.688 & 0.000 & 19.309 \\
\hline Intercept & 7.777 & 2.942 & 0.008 & $2.386 \mathrm{E} 3$ \\
\hline \multicolumn{3}{|c|}{-2 Log likelihood=101.274 } & \multicolumn{2}{|c|}{ Cox \& Snell $R$ Square $=0.663$} \\
\hline
\end{tabular}

Data source: software output

From the model overall, R2 after adjusted can reach to0.906, it indicates that the model overall fit is better, and independent variables can be used to explain the dependent variable. From the various factors, there are age, education level, family income, the amount of annual consumption ginseng, ginseng brand and quality certification marks, and these 6 variables through the significant level of 0.05 tests. From the model estimation results, they can be obtained the following conclusions:

The age of consumer affects the behavior of purchase ginseng brand. From the model results, the age of consumer has significantly affected on their purchase brand ginseng behavior, but the direction is positive. This shows that as consumers age, consumers tend to buy brand ginseng more, and this is consistent with the hypothesis before.

The education level of consumers has effect on the behavior of purchase brand ginseng. From the model results, the higher the education level of consumers, the tendency of purchase brand ginseng will be greater. This shows that the higher the level of education, people more emphasis on packaging, quality and other factors in its purchase ginseng products or consumer, and they are willing to buy brand ginseng. At the same time, the higher consumer education, the income may be higher, and this explains its purchase intention to a certain extent.

The family income of consumers affects their consumption tendency. From the model results, the higher the income of consumer families, the possibility of buying brand ginseng is more. Therefore, the consumer's income is higher, the high standard of living, so the choices of consumer 
goods, including ginseng products, people more wills to choose high-quality products, so tend to buy brand ginseng products.

The amount of consumers' annual consumption ginseng. From the model results, the amount of annual consumption of ginseng consumers has significantly affected on the purchase behavior. Every year consumers buy more money spent ginseng, this shows that health and nutritional function of ginseng are more recognized. In this premise, the more willing to buy good quality brand ginseng is well understood.

Whether the consumers understand the quality certification marks on effect of the purchase brand ginseng. From the model results, whether consumers understand the quality safety certification mark on the significantly effect of their purchase behavior, consumers understand the quality certification marks is very helpful for the quality assurance about brand ginseng. After understanding the brand ginseng has quality certification marks, consumers will increase on the probability of the choice of brand ginseng.

Whether consumers understand the ginseng brand on the influence of purchase behavior. From the model results, whether consumers understand ginseng brand on the significant impact of buying brand, people can be a good understanding on these factors, only to understand the ginseng brand, consumers will choose the relatively high price of the brand ginseng. Because consumers do not understand the ginseng brand, rational consumers do not spend a relatively high price to buy non brand products with on difference in appearance of ginseng.

Anther variables. In addition to the above variables of significantly influence, the consumer's gender, marital status, number of family members, purchase purpose and product price do not be cared by model checking.

\section{Conclusions and Policy Recommendations}

Reasonable planning is essential for the development of ginseng brand, so it is necessary to build a ginseng brand development system from the strategic level. The brand strategic system is divided into prospective planning, management planning and culture planning. In the management plan, the system also includes import strategy and business strategy, in which import strategy includes the brand awareness and brand positioning; business strategy includes brand extension and brand maintenance. This paper constructs ginseng brand strategy system from the aspects of three plantings.

Vision planning. The prospective planning of ginseng brand contains two aspects, in which one is the full development of the domestic consumer market, so that consumers fully understand the brand ginseng value and ginseng culture through the cultivation and development of the domestic market; the two is to establish and improve the brand awareness of Chinese ginseng in the international market, especially Changbai Mountain area ginseng brands in domestic main producing areas. The Chinese ginseng is created a famous brand in the international market, breaking South Korea, Europe and the United States and other countries on the monopoly of high-end ginseng products, and foreign consumers first thought Chinese ginseng in the consumer, so that it can help to improve Chinese ginseng in the market competitiveness.

Management planning. Ginseng brand management planning can be from two aspects, one is the import strategy, and another is business strategy. Import strategy refers to the analysis results of the early brand, determining the ginseng brand market positioning, target customer groups, product prices and other factors; according to the actual situation and the external environment of the industrial development, business strategy mainly refers to the appropriate program to enhance the brand competitive force, including brand maintenance, brand extension and so on. The positioning ginseng brand mainly refers to the health care. Target customers positioning has the white-collar class of the stable income source, the class of general population have received a good education, they are in-depth understanding of health concept and pay attention to their and their family's health with health awareness, and they are consumer willingness and ability to buy for ginseng products. Brand maintenance is that when the external environment changes, the brand maintains market position and its vitality to carry out marketing activities, including product quality, appearance, law 
and so on, in which the quality is the premise, it is the guarantee for the sustainable development of the health industry, but also carry out the basis of other activities. The appearance maintenance is different from other products unique personality in order to shape the brand. Legal maintenance mainly refers to protect the brand itself from the angle of law. Brand extension is defined by product line extension, vice product line extension and licensing system, making the brand get sustainable development, so that the new products can successfully seize the market with existing successful brand reputation.

Cultural planning. Our country has a unique ginseng culture, but also is ginseng suzerain and ginseng powers. In the world, the scope of ginseng cultivation area is the largest, it is the highest yield, and the main producing areas are in Jilin province. According to the cultural relic research and historical records, people found that the application of ginseng is at least four thousand years in China, the history of ginseng acquisition is at least two thousand and five hundred years, and ginseng cultivation history is more than two thousand years. Especially in Jilin, it has a profound cultural story about ginseng. For a long time, Changbai Mountain area residents who dug into the mountains formed a unique folk customs, including various taboos, professional language, behavior standard, technical specification, special tools and a series of rich content. So, in the ginseng brand culture planning, people should highlight the special Chinese ginseng and suzerain status, and let ginseng consumers understand the birthplace of the ginseng culture in China's Jilin, Changbai Mountain area ginseng in Jilin is really high quality and authentic ginseng.

\section{References}

[1] Zigang Yang. The implementation of brand strategy to revitalize the ginseng industry of Jilin [J]. Business research, 2014 (21): 89-90.

[2] Haitao Li. Study of the leapfrog development of Jilin province ginseng industry. Chinese Agricultural Science Bulletin, 2015, 26 (22): 238-243.

[3] Jun Wang. Empirical analysis of the consumer to purchase high-quality security ginseng products will and its affecting factors. Chinese rural economy, 2014(5): 35-42.

[4] Ce Zheng et al. Study on Jilin Province ginseng brand strategy based on SWOT analysis method. The seventh Jilin province science and technology conference, 2013: 445-446.

[5] Mohan Feng. Jilin Province "Changbai Mountain Ginseng” regional brands to create problems and countermeasures. Research enterprise, 2013(5): 54-56.

[6] Zheng Sun. Jilin Province ginseng industry development analysis [J]. Anhui Agricultural Sciences, 2014, 36(23): $119-121$.

[7] Chengrong Wang. Study on the evaluation and management of brand value [D]. Journal of Huazhong University of Science and Technology, 2015: 1-12.

[8] Chengrong Wang. The improvement and innovation of brand value social evaluation method [J]. Management review, 2015 (1): 47-52.

[9] Chengrong Wang. On the origins of brand value and its composition [J]. Business research, 2014(9): 7-10.

[10] Lu Zheng. Study on the strategy of China agricultural products brand building [D]. Jiangnan University, 2014: 1-9.

[11] Lulu Meng. The present situation and thinking of the ginseng industry in Jilin province [J]. Social science front, 2014(12): 112-113.

[12] Keller K.L. Conceptualizing measuring and managing customer-based equity [J]. Journal of Marketing, 2014(81): 1-12. 